\title{
Spectrum of the volume operator in quantum gravity
}

\author{
R. Loll ${ }^{1}$ \\ Sezione INFN di Firenze, Largo E. Fermi 2, I-50125 Florence, Italy \\ and Max-Planck-Institut für Gravitationsphysik, Schlaatzweg 1, D-14473 Potsdam, Germany
}

Received 16 November 1995; accepted 1 December 1995

\begin{abstract}
The volume operator is an important kinematical quantity in the non-perturbative approach to four-dimensional quantum gravity in the connection formulation. We give a general algorithm for computing its spectrum when acting on four-valent spin network states, evaluate some of the eigenvalue formulae explicitly, and discuss the role played by the Mandelstam constraints.
\end{abstract}

\section{Introduction}

The volume operator has emerged as an important quantity in the kinematics of $(3+1)$-dimensional quantum gravity in the loop representation. It is the quantum analogue of the classical volume function, measuring the volume of three-dimensional spatial regions. Although not an observable of the pure gravity theory (in the sense of commuting with the gravitational Hamiltonian), it has an immediate physical interpretation, and becomes a genuine observable if the spatial regions are defined intrinsically by additional matter fields, for example, the constant-value surfaces of a scalar field variable. Moreover, the volume is a relatively simple function of the dreibein variable $E$, of the canonically conjugate pair $(A, E)$, where $A \in \mathcal{A}$ is the (complex) $S U(2)$-Ashtekar connection on the three-space $\Sigma$.

The significance of the volume operator for the quantum theory derives from the fact that its eigenfunctions span the kinematical quantum state space of functions on $\mathcal{A} / \mathcal{G}$, the space of connections modulo gauge transformations, and that these functions are

\footnotetext{
${ }^{1}$ Supported by the European Human Capital and Mobility program "Constrained Dynamical Systems".
} 
known, at least in principle [1]. They are the so-called spin network states, which can be expressed as appropriately (anti-) symmetrized linear combinations of certain Wilson loop functions (traces of holonomies of the connection variable $A$ ). This, as well as the spectral discreteness of the volume operator, was first pointed out in [2].

An obvious task at this stage is the actual computation of its spectrum. We will describe in the following a general algorithm for computing the volume spectrum on four-valent spin network states, that is, spin networks made up of Wilson loop states with no more than four line segments meeting at each loop intersection. The eigenvalues of several classes of such states will be computed explicitly. We have shown previously [3] that the spectrum in the three-valent case is identically zero, thereby correcting a computational error in [2], where a non-vanishing spectrum was derived. (Because of the algebraic structure of the volume operator it is clear a priori that loop states with valence less than three are annihilated.)

Our calculations will take place in a lattice-regularized framework which we have been advocating elsewhere as an appropriate tool for approximating the quantum Hamiltonian dynamics of gravity in the loop approach [4]. However, to our understanding the form of the lattice operator coincides with that of the continuum formulations [5,6] (when restricted to the subset of states that can be realized on a three-dimensional cubic lattice), and therefore our results are equally valid in those cases. Since the discussion of the volume operator is largely insensitive to the signature of space-time and the particular form of the Hamiltonian, we will for simplicity work within the real, $S U(2)$ setting. We only remind the reader that there is a version of Ashtekar's gravity based on a real canonical variable pair $(A, E)$, as recently discussed in [7] (where, however, the Hamiltonian assumes a more complicated form than in the complex formulation).

As part of the spectrum calculation, we will have to address the issue of overcompleteness of the spin network states, which is a familiar feature of complete sets of gauge-invariant functions on a space $\mathcal{A}$ of gauge potentials. We will argue that it is necessary to eliminate this over-completeness in order to derive the correct spectrum for the volume operator.

In the next section, we will recall the construction of the classical volume function and the discretized quantum volume operator on the lattice. In Section 3, we derive a general expression for the matrix elements of the volume operator on four-valent spin network states, which is then illustrated by explicitly calculating some of the spectral formulae in Section 4. In Section 5 we summarize and discuss our results.

\section{Defining the volume operator}

In order to fix the notation, we will first summarize the main ingredients of the customary Hamiltonian lattice formulation for gauge theories [8]. Our lattice is a cubic $N \times N \times N$ lattice, with periodic boundary conditions, i.e. the topology of a three-torus. The basic operators associated with each lattice link $l$ are an $S U(2)$-link holonomy $\hat{V}$, together with its inverse $\hat{V}^{-1}$, and a pair of canonical momentum operators $\hat{p}_{i}^{+}$and 
$\hat{p}_{i}^{-}$, where $i$ is an adjoint index. The operator $\hat{p}_{i}^{+}(n, \hat{a})$ is based at the vertex $n$, and is associated with the link $l$ oriented in the positive $\hat{a}$-direction. By contrast, $\hat{p}_{i}^{-}\left(n+\hat{l}_{\hat{a}}, \hat{a}\right)$ is based at the vertex displaced by one lattice unit in the $\hat{a}$-direction, and associated with the inverse link $l^{-1}(\hat{a})=l(-\hat{a})$. The wave functions are elements of $\otimes_{l} L^{2}(S U(2), d g)$, with the product taken over all links, and $d g$ is the Haar measure. The basic commutators are

$$
\begin{aligned}
{\left[\hat{V}_{A}^{B}(n, \hat{a}), \hat{V}_{C}^{D}(m, \hat{b})\right] } & =0, \\
{\left[\hat{p}_{i}^{+}(n, \hat{a}), \hat{V}_{A}^{C}(m, \hat{b})\right] } & =-\frac{i}{2} \delta_{n m} \delta_{\hat{a} \hat{b}} \tau_{i A}^{B} \hat{V}_{B}^{C}(n, \hat{a}), \\
{\left[\hat{p}_{i}^{-}(n, \hat{a}), \hat{V}_{A}^{C}(m, \hat{b})\right] } & =-\frac{i}{2} \delta_{n m} \delta_{\hat{a} \hat{b}} \hat{V}_{A}^{B}(n, \hat{a}) \tau_{i B}{ }^{C}, \\
{\left[\hat{p}_{i}^{ \pm}(n, \hat{a}), \hat{p}_{j}^{ \pm}(m, \hat{b})\right] } & = \pm i \delta_{n m} \delta_{\hat{a} \hat{b}} \epsilon_{i j k} \hat{p}_{k}^{ \pm}(n, \hat{a}), \\
{\left[\hat{p}_{i}^{+}(n, \hat{a}), \hat{p}_{j}^{-}(m, \hat{b})\right] } & =0,
\end{aligned}
$$

where $\epsilon_{i j k}$ are the structure constants of $S U(2)$. In terms of an explicit parametrization by four complex parameters $\alpha_{i}, i=0, \ldots, 3, \sum_{i} \alpha_{i}^{2}=1$, the operators for a single link $(n, \hat{a})$ are given by

$$
\begin{aligned}
& \hat{V}_{A}^{B}=\left(\begin{array}{cc}
\alpha_{0}+i \alpha_{1} & \alpha_{2}+i \alpha_{3}, \\
-\alpha_{2}+i \alpha_{3} & \alpha_{0}-i \alpha_{1}
\end{array}\right)=\alpha_{0} 1+\sum_{i=1}^{3} \alpha_{i} \tau_{i}, \\
& \hat{p}_{1}^{ \pm}=\frac{i}{2}\left(\alpha_{1} \partial_{0}-\alpha_{0} \partial_{1} \pm \alpha_{3} \partial_{2} \mp \alpha_{2} \partial_{3}\right), \\
& \hat{p}_{2}^{ \pm}=\frac{i}{2}\left(\alpha_{2} \partial_{0} \mp \alpha_{3} \partial_{1}-\alpha_{0} \partial_{2} \pm \alpha_{1} \partial_{3}\right), \\
& \hat{p}_{3}^{ \pm}=\frac{i}{2}\left(\alpha_{3} \partial_{0} \pm \alpha_{2} \partial_{1} \mp \alpha_{1} \partial_{2}-\alpha_{0} \partial_{3}\right),
\end{aligned}
$$

where in the first line we have defined the three $\tau$-matrices. In the continuum theory, the classical expression for the volume of a spatial region $\mathcal{R} \subset \Sigma$ is given by

$$
\mathcal{V}(\mathcal{R})=\int_{\mathcal{R}} d^{3} x \sqrt{\operatorname{det} g}=\int_{\mathcal{R}} d^{3} x \sqrt{\frac{1}{3 !}\left|\epsilon_{a b c} \epsilon^{i j k} E_{i}^{a} E_{j}^{b} E_{k}^{c}\right|},
$$

where $E_{i}^{a}$ are the dreibein variables introduced earlier (corresponding to the generalized electric fields in a gauge theoretic language). Taking into account the continuum limit of the classical lattice variables,

$$
p_{i}^{ \pm}(n, \hat{a}) \stackrel{a \rightarrow 0}{\longrightarrow} a^{2} \tilde{E}_{i}^{u}(n)+O\left(a^{3}\right),
$$

as the lattice spacing $a$ goes to zero, we define the lattice analogue of (2.3) as

$$
\begin{aligned}
& \mathcal{V}_{\text {latt }}= \\
& \sum_{n \in \mathcal{R}} \sqrt{\frac{1}{48}\left|\epsilon_{a b c} \epsilon^{i j k}\left(p_{i}^{+}(n, \hat{a})+p_{i}^{-}(n, \hat{a})\right)\left(p_{j}^{+}(n, \hat{b})+p_{j}^{-}(n, \hat{b})\right)\left(p_{k}^{+}(n, \hat{c})+p_{k}^{-}(n, \hat{c})\right)\right|} .
\end{aligned}
$$


For consistency, we have averaged over the momenta of both orientations ${ }^{2}$. The translation of this expression to the quantum theory is not well defined a priori, because of the presence of both the modulus and the square root. However, since both the $\hat{p}^{ \pm}$and therefore also the operators

$$
\begin{aligned}
\hat{D}(n):= & \frac{1}{8} \epsilon_{a b c} \epsilon^{i j k}\left(\hat{p}_{i}^{+}(n, \hat{a})+\hat{p}_{i}^{-}(n, \hat{a})\right)\left(\hat{p}_{j}^{+}(n, \hat{b})+\hat{p}_{j}^{-}(n, \hat{b})\right) \\
& \times\left(\hat{p}_{k}^{+}(n, \hat{c})+\hat{p}_{k}^{-}(n, \hat{c})\right)
\end{aligned}
$$

are self-adjoint, we may go to a Hilbert space basis of simultaneous eigenfunctions of all the $\hat{D}(n)$ and define the operator

$$
\hat{V}_{\text {latt }}=\sum_{n} \sqrt{\frac{1}{3 !}|\hat{D}(n)|}
$$

through the square roots of the moduli of the eigenvalues of the $\hat{D}(n)$ in that basis. (Note that no operator ordering problem occurs in the definition of $\hat{D}(n)$.) As already mentioned in the introduction, the diagonalization of the volume operator is most easily achieved starting from a set of spin network states on the lattice. These are certain (anti-) symmetrized, real linear combinations of Wilson loops. (A Wilson loop on the lattice is a gauge-invariant function of the form $\operatorname{Tr} V\left(l_{1}\right) V\left(l_{2}\right) \ldots V\left(l_{k}\right)$, where $\gamma=l_{1} \circ l_{2} \circ \ldots \circ l_{k}$ is a closed loop of lattice links.) A spin network associates a positive "occupation number" with each lattice link, counting the number of (unoriented) flux lines of basic spin- $\frac{1}{2}$ representations along the link, and also keeps track of the way in which those flux lines are contracted gauge-invariantly at the vertices (see Ref. [1] for more details).

The operators $\hat{D}(n)$ have a particularly simple action on spin networks, because they do not change their support (in terms of the flux line numbers). Thus only finitedimensional rearrangements occur within each subset of states sharing the same occupation numbers, and the diagonalization of $\hat{\mathcal{V}}_{\text {latt }}$ can be performed separately in these finite-dimensional eigenspaces [2]. In this respect, the structure of the volume operator is much simpler than that of the Hamiltonian operator, which (at least on the lattice) changes the support of Wilson loops [4]. Since an operator $\hat{D}(n)$ acts only on links adjacent to the vertex $n$, and neighbouring $\hat{D}(n)$ 's commute, it is sufficient to study its action on spin networks locally around a single vertex. This will be the subject of the next section.

\section{Deriving the spectrum on four-valent spin networks}

Because of the cubic geometry of the lattice, the spin networks that can be defined on it are at most six-valent.

\footnotetext{
${ }^{2}$ This choice does not alter the conclusions of [3], where the unaveraged operator was used. The eigenvalues reported in [3] are merely changed by a constant overall factor, e.g., $c=\frac{1}{4}$ for four-valent intersections.
} 


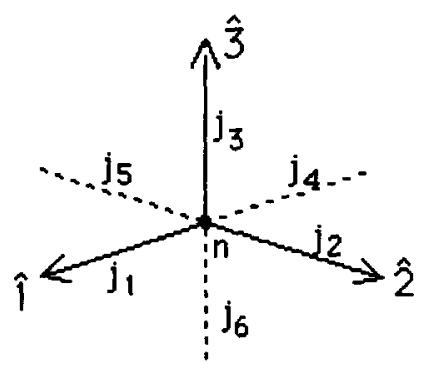

Fig. 1.

Fig. 1 illustrates our labelling of the link directions meeting at a vertex $n$. One ingredient in describing a spin network locally around $n$ is a 6-tuple $j$ of integers $j_{i} \geqslant 0$ giving the occupation numbers $\left(j_{1}, \ldots, j_{6}\right)$ of the links

$$
\begin{aligned}
& ((n, \hat{1}),(n, \hat{2}),(n, \hat{3}) ;(n,-\hat{1}),(n,-\hat{2}),(n,-\hat{3})) \\
& \quad \equiv((n, \hat{1}),(n, \hat{2}),(n, \hat{3}) ;(n-\hat{1}, \hat{1}),(n-\hat{2}, \hat{2}),(n-\hat{3}, \hat{3}))
\end{aligned}
$$

intersecting at $n$. Since the flux lines are to be contracted at the vertex, their sum $j:=\sum_{i=1}^{6} j_{i}$ is an even integer. Next one has to specify how the $j$ flux lines are joined pairwise at $n$ to ensure gauge-invariance.

By convention we may join a flux line along the positive $\hat{\mathbf{1}}$-direction, say, only to a flux line from one of the other five links, and not from the same link (i.e. we forbid "retracings"). This leads to a constraint on the occupation numbers: any $j_{i}$ has to be equal to or smaller than the sum of the remaining $j_{k}$, i.e. $j_{i} \leqslant \sum_{k \neq i} j_{k}$. Given a contraction of the flux lines at the vertex, the spin network consists of a weighted linear combination of $j_{1} ! j_{2} ! j_{3} ! j_{4} ! j_{5} ! j_{6}$ ! Wilson loops corresponding to all possible permutations of flux lines associated with each of the six links. The weight factors are given by $(-1)^{(P+N)}$, where $P$ is the parity of the flux line permutation and $N$ the number of closed loops in a multiple Wilson loop that is obtained by arbitrarily completing the local link configuration around the vertex $n$. The relative weights of the set of multiple Wilson loops thus obtained is independent of such an extension. To obtain a complete spin network state, this (anti-) symmetrization of course has to be performed around every vertex contained in the state.

Since the diagonalization of the volume operator is algebraically rather involved, we will restrict ourselves to the simplest non-trivial case, namely that of spin networks with at most four-valent intersections. This is consistent since the volume operator maps the set of such states into itself. In a previous paper we have shown that spin networks are annihilated locally by $\hat{D}(n)$ at trivalent intersections, and more generally at intersections for which there exists only a single, unique contraction of flux lines [3]. From our calculations on four-valent spin networks below one recognizes this as part of a general pattern, namely that eigenvalues occur in pairs of opposite sign.

Without loss of generality we may restrict our attention to four-valent vertices with 


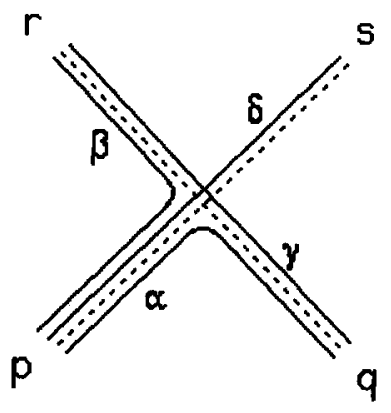

Fig. 2.

occupation numbers of the form $j=(p, q, r ; s, 0,0)$, with $p \geqslant q \geqslant r \geqslant s>0$. A moment's consideration reveals that the number of spin network states one may construct from this link configuration is $(x+1)(x+2) / 2$, where $x=\operatorname{Min}\left\{s, \frac{1}{2}(-p+q+r+s)\right\}$. However, these spin networks are not all linearly independent, due to the existence of the so-called Mandelstam constraints (see, for example, Ref. [9]). It turns out that the number of linearly independent four-valent spin networks is $x+1$. Thus it grows only linearly in $x$, whereas the total number of states is proportional to $x^{2}$. (It is a question of semantics whether by "spin networks" one means the full set of (anti-) symmetrized states as introduced above or only an independent, already orthogonalized basis set-we have been using it in the former sense.)

An alternative way of parametrizing the occupation numbers is given by four integers $(\alpha, \beta, \gamma, \delta)$, where $p=\alpha+\beta+\delta, q=\alpha+\gamma, r=\beta+\gamma$, and $s=\delta$. (See Fig. 2; by drawing $\alpha$ etc. as connected pieces of incoming and outgoing flux lines we do not mean to indicate that the associated spin network states will share the same routings at the intersection; it merely gives us another way of labelling a flux line configuration.) Note that this transformation is invertible. The variable change is useful because of the following construction. Abandon for the moment the restrictions $p \geqslant q \geqslant r \geqslant s$, keeping however the inequalities $p \leqslant(q+r+s), q \leqslant(p+r+s), r \leqslant(p+q+s)$, $s \leqslant(p+q+r)$ and the condition $(p+q+r+s) \bmod 2=0$. Given a fixed $x \geqslant 1$, we define the "fundamental link configuration" to be the one with minimal total occupation number $(p+q+r+s)$. For this configuration we obviously have $(p, q, r, s)=(x, x, x, x)$, or $(\alpha, \beta, \gamma, \delta)=(0,0, x, x)$. Now observe that every allowed link configuration with the same $x$ can be obtained by adding 4-tuples of non-negative integers $(\Delta \alpha, \Delta \beta, \Delta \gamma, \Delta \delta)$ to $(\alpha, \beta, \gamma, \delta)=(0,0, x, x)$. The converse is not true, because adding such a quadruplet may change $x$.

Our aim is to derive general formulae for the eigenvalues of $\hat{D}(n)$ on independent spin networks for fixed $x$. This can be achieved in a series of steps. Starting from a link configuration $(\alpha, \beta, \gamma, \delta)=(0,0, x, x)$, one extends it arbitrarily to obtain a multi-loop, and then determines the corresponding set of $(x+1)(x+2) / 2$ spin networks. A subset of $x+1$ linearly independent states can be easily determined using, for example, the relations derived in [9]. Note that, due to the total (anti-) symmetry of the spin network 
states, loop configurations containing retracings on any of the links meeting at $n$ can be set identically to zero. Next one computes the action of $\hat{D}(n)$ on the independent states, thus obtaining a $(x+1) \times(x+1)$-matrix, which one then diagonalizes. It is equivalent and much easier to compute the action of $\hat{D}(n)$ on a single representative of the permutation equivalence class of Wilson loop states that make up a given spin network, and then check which permutation equivalence classes the resulting Wilson loop functions lie in. In all of this, one must remember to keep track of the weight factors $(-1)^{(P+N)}$. A useful identity in evaluating the action of $\hat{D}(n)$ is

$$
\epsilon^{i j k}\left(\tau_{i}\right)_{A}^{B}\left(\tau_{j}\right)_{C}^{D}\left(\tau_{k}\right)_{E}^{F}=2\left(\delta_{A}^{D} \delta_{E}^{B} \delta_{C}^{F}-\delta_{A}^{F} \delta_{C}^{B} \delta_{E}^{D}\right)
$$

All of the above steps may be implemented using algebraic computing programs, like Mathematica. In the derivation of the eigenvalue formulae at constant $x$ it is crucial to observe that it is sufficient to know the action of $\hat{D}(n)$ on a small number of link configurations $(\alpha, \beta, \gamma, \delta)$ (or rather their associated spin networks) "close" to the fundamental one, $(0,0, x, x)$, as we will now proceed to explain.

Let us adopt the shortcut described above for deriving the action of $\hat{D}(n)$ on spin networks by their action on Wilson loop representatives. Note that going from the link configuration $(0,0, x, x)$ to $(1,0, x, x)$, say, at the level of these Wilson loop representatives may be represented by adding a single closed loop "containing $\alpha$ " (for an explicit example, see Section 4). It is then easy to repeat the steps outlined above, and determine the matrix elements of $\hat{D}(n)$. Call the matrices obtained in this way $M(0,0, x, x)$ and $M(1,0, x, x)$ respectively. Using the explicit action of $D(n)$ on Wilson loop states, one may then prove that any $M(\alpha, 0, x, x), \alpha=2,3, \ldots$, can be computed via

$$
M(\alpha, 0, x, x)=M(0,0, x, x)+\alpha(M(1,0, x, x)-M(0,0, x, x)) .
$$

Similar relations exist for other link configurations, and one can derive a general formula expressing the matrix representation of $\hat{D}(n)$ acting on the set of spin networks associated with an arbitrary link configuration $(\alpha, \beta, \gamma, \delta)$,

$$
\begin{aligned}
M_{x}(\alpha, \beta, \gamma, \delta)= & (1-\gamma+x)(1-\delta+x)\left((1-\alpha)(1-\beta) M_{x}(0,0, x, x)\right. \\
& +\alpha(1-\beta) M_{x}(1,0, x, x) \\
& \left.+(1-\alpha) \beta M_{x}(0,1, x, x)+\alpha \beta M_{x}(1,1, x, x)\right) \\
& +((\gamma-x)(1-\delta+x)+(1-\gamma+x)(\delta-x)) \\
& \times\left((1-\alpha)(1-\beta) M_{x}(0,0, x+1, x)\right. \\
& +\alpha(1-\beta) M_{x}(1,0, x+1, x) \\
& \left.+(1-\alpha) \beta M_{x}(0,1, x+1, x)+\alpha \beta M_{x}(1,1, x+1, x)\right),
\end{aligned}
$$

where we have introduced a subscript $x$ to indicate that the matrix elements of $M$ refer to sets of spin networks with fixed $x$. Formula (3.2) is of course a special case of (3.3). In the derivation of (3.3) we have taken into account that $M_{x}(0,0, x+1, x)=$ $M_{x}(0,0, x, x+1), M_{x}(1,0, x+1, x)=M_{x}(1,0, x, x+1)$, etc. 
(a)

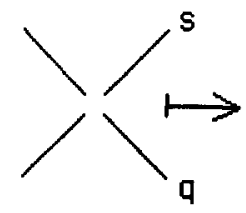

(b)

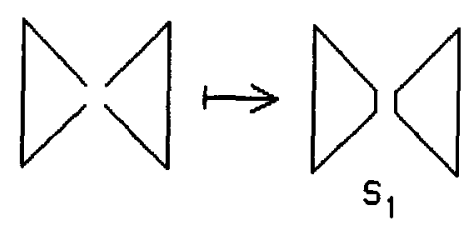

(c)

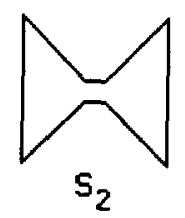

Fig. 3.

Given this expression, it is straightforward to establish the explicit eigenvalue formulae for a given $x$. To illustrate our method, we will in the next section discuss the case $x=1$ in some detail, and also give the eigenvalue formulae for $x=2$ and 3.

\section{Computation of the spectrum for small $x$}

Recall first that, for $x=0$, following [3], all eigenvalues of the volume operator vanish identically. Let us therefore turn to the case $x=1$. The fundamental link configuration is given by $(\alpha, \beta, \gamma, \delta)=(0,0,1,1)$ or, equivalently, $(p, q, r, s)=(1,1,1,1)$, Fig. 3a.

Now close up the external ends of the four links in some way, as shown schematically in Fig. 3b. There are then three spin networks, $S_{1}, S_{2}$ and $S_{3}$, obtained by connecting the internal ends of the links meeting at the vertex $n$ in various ways (Fig. $3 \mathrm{c}$ ). Since the links are singly occupied, no permutations have to be taken into account. The Mandelstam identity for this set of spin networks is $S_{1}-S_{2}-S_{3}=0$. We choose as an independent set the states $S_{1}$ and $S_{2}$. One then computes the matrix representation for $\hat{D}(n)$ on these states, which is given by

$$
M_{1}(0,0,1,1)=\frac{3 i}{8}\left(\begin{array}{ll}
1 & -2 \\
2 & -1
\end{array}\right) \text {. }
$$

According to our reasoning of the previous section, we have to compute another seven matrices $M_{1}$.

Consider next the link configuration $(\alpha, \beta, \gamma, \delta)=(1,0,1,1)$. This means adding two links to the previous configuration, as illustrated in Fig. $4 \mathrm{a}$ and Fig. 4b. The analogue of Fig. $3 c$ is shown in Fig. 4c.

The three configurations $W_{1}, W_{2}$ and $W_{3}$ are now not spin networks themselves (because no (anti-) symmetrization has been performed), but can be considered as Wilson loop representatives of permutation equivalence classes, as explained earlier. For the corresponding spin networks $S_{1}^{\prime}, S_{2}^{\prime}$ and $S_{3}^{\prime}$, by construction $S_{1}^{\prime}-S_{2}^{\prime}-S_{3}^{\prime}=0$ continues to hold, and one computes

$$
M_{1}(1,0,1,1)=\frac{3 i}{8}\left(\begin{array}{ll}
2 & -3 \\
4 & -2
\end{array}\right)
$$


(a)

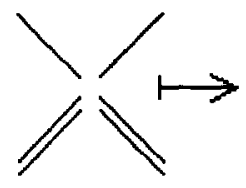

(b)

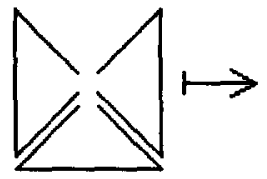

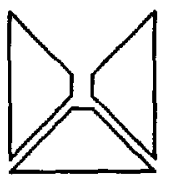

$W_{1}$ (c)

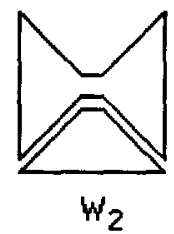

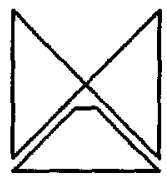

$w_{3}$

Fig. 4.

in the basis $\left\{S_{1}^{\prime}, S_{2}^{\prime}\right\}$. Proceeding similarly with the other relevant link configurations, one obtains for (3.3)

$$
M_{1}(\alpha, \beta, \gamma, \delta)=\frac{3 i}{8}\left(\begin{array}{cc}
(1+\alpha)(1+\beta) & -(1+\beta)(\alpha+\gamma+\delta) \\
(1+\alpha)(\beta+\gamma+\delta) & -(1+\alpha)(1+\beta)
\end{array}\right),
$$

from which one computes the eigenvalues of $\hat{D}(n)$ on link configurations $(\alpha, \beta, \gamma, \delta)$ as

$$
\pm \frac{3}{8} \sqrt{(1+\gamma+\delta)(1+\alpha)(1+\beta)(1+\alpha+\beta+\gamma+\delta)} .
$$

Note that we have used the identity $(\gamma-1)(\delta-1)=0$, valid for $x=1$. Going back to the notation $(p, q, r, s)$ for link configurations, with $p \geqslant q \geqslant r \geqslant s>0$, one finds for the first few configurations the following eigenvalues:

\begin{tabular}{ccccccc}
\hline$(p, q, r, s)$ & $(1,1,1,1)$ & $(2,2,1,1)$ & $(3,2,2,1)$ & $(3,3,1,1)$ & $(3,3,3,1)$ & $(4,2,2,2)$ \\
eigenvalues & $\pm \frac{3}{8} \sqrt{3}$ & $\pm \frac{3}{2 \sqrt{2}}$ & $\pm \frac{3 \sqrt{5}}{4}$ & $\pm \frac{3}{8} \sqrt{15}$ & $\pm \frac{3 \sqrt{3}}{2}$ & $\pm \frac{3 \sqrt{3}}{2}$ \\
\hline
\end{tabular}

Similarly, we have computed the eigenvalues of $\hat{D}(n)$ for $x=2$. For each link configuration there are six spin network states, out of which three are linearly independent. For the matrix $M_{2}(\alpha, \beta, \gamma, \delta)$, there is one zero-eigenvalue and a pair of non-vanishing ones,

$$
\begin{aligned}
& \pm \frac{3}{4 \sqrt{2}}[-(2+\alpha)(2+\beta)(2+\alpha+\beta) \\
& \quad+(\gamma+\delta-2)(\alpha+\beta+\gamma+\delta)(2 \alpha \beta+3 \alpha+3 \beta+4)]^{1 / 2} .
\end{aligned}
$$

In principle, formula (3.3) can be used also for the unreduced matrices, i.e. before the linearly dependent spin networks are eliminated. However, it is unfortunately not true that such states automatically have vanishing eigenvalues. Of course, if complex eigenvalues occur it is clear that they must correspond to spin networks that vanish modulo the Mandelstam constraints. But even non-zero, real eigenvalues occur for these spurious eigenvectors. (This happens, for example, in the case $x=3$.) Again, we give a list of the first few eigenvalues corresponding to the matrix $M_{2}(\alpha, \beta, \gamma, \delta)$ : 


\begin{tabular}{ccccccc}
\hline$(p, q, r, s)$ & $(2,2,2,2)$ & $(3,3,2,2)$ & $(4,3,3,2)$ & $(4,4,2,2)$ & $(4,4,4,2)$ & $(5,3,3,3)$ \\
eigenvalues & $\pm \frac{3 \sqrt{3}}{2}$ & $\pm \frac{3 \sqrt{13}}{2 \sqrt{2}}$ & $\pm \frac{9 \sqrt{3}}{2 \sqrt{2}}$ & $\pm \frac{3}{2} \sqrt{11}$ & $\pm \frac{9}{2} \sqrt{3}$ & $\pm \frac{9}{2} \sqrt{3}$ \\
\hline
\end{tabular}

For higher $x$, the algebra becomes progressively more complicated, but no problems occur in principle in determining the eigenvalues. Here are the eigenvalues of the matrix $M_{3}(\alpha, \beta, \gamma, \delta)$ for the case $x=3$, which come in two pairs of opposite sign, namely,

$$
\pm \frac{3}{8} \sqrt{I_{1} \pm \sqrt{I_{2}}}
$$

where

$$
\begin{aligned}
I_{1}= & 5 \mu(\alpha+2)(\beta+2) \nu-3 \mu(\mu+2)+3(\alpha+\beta+6)(\gamma+\delta)+3(\alpha \beta-11) \\
I_{2}= & 16(\mu+2)^{2} \nu^{2}(\alpha+1)(\beta+1)(\alpha+3)(\beta+3)+5(\mu+2)^{2} \nu^{2}\left(5 \alpha^{2}\right. \\
& \left.+5 \beta^{2}+8 \alpha+8 \beta-6 \alpha \beta\right) \\
& +64(\mu+2)^{2} \nu^{2}-64(\mu+2) \nu^{2}(\alpha+2)^{2}(\beta+2)^{2} \\
& \left.+12(\mu+2)^{3}\left(10(\alpha+2)(\beta+2)-3(\alpha+2)^{2}-3(\beta+2)^{2}\right)\right) \\
& +4(13 \mu+15) \nu(\alpha+2)^{2}(\beta+2)^{2} \\
& -6 \nu^{2}(\alpha+2)(\beta+2)\left(5 \alpha^{2}+5 \beta^{2}+48 \alpha+48 \beta+244\right) \\
& +36 \nu^{2}\left(2 \alpha^{3}+17 \alpha^{2}+44 \alpha+2 \beta^{3}+17 \beta^{2}+44 \beta+72\right) \\
& -94 \nu(\alpha+2)^{2}(\beta+2)^{2}(\alpha+\beta+10) \\
& -6 \nu(\alpha+2)(\beta+2)\left(5 \alpha^{3}+54 \alpha^{2}+316 \alpha+5 \beta^{3}+54 \beta^{2}+316 \beta+1152\right) \\
& +108 \nu\left((\alpha+2)^{2}(a+4)^{2}+(\beta+2)^{2}(\beta+4)^{2}\right) \\
& +36(\alpha+4)^{3}(\alpha+2)^{2}+36(\beta+4)^{3}(\beta+2)^{2} \\
& +18(\alpha+4)(\beta+4)(\alpha+2)^{2}(\beta+2)^{2}-960(\alpha+2)(\beta+2) \\
& +9(\alpha+2)(\beta+2)(\alpha+4)(\beta+4)\left(\alpha^{2}-26 \alpha+\beta^{2}-26 \beta-112\right) \\
& +6(\alpha+2)(\beta+2)\left((\alpha+2)\left(-5 \alpha^{2}+16 \alpha+64\right)\right. \\
& \left.+(\beta+2)\left(-5 \beta^{2}+16 \beta+64\right)\right)
\end{aligned}
$$

with the abbreviations $\mu=\alpha+\beta+\gamma+\delta$ and $\nu=\gamma+\delta-4$. For small occupation numbers $(p, q, r, s)$, some explicit eigenvalues are given by

\begin{tabular}{lcccc}
\hline$(p, q, r, s)$ & $(3,3,3,3)$ & $(4,4,3,3)$ & $(5,4,4,3)$ & $(5,5,5,3)$ \\
eigenvalues & $\pm \frac{9}{8} \sqrt{19 \pm 16}$ & $\pm \frac{9}{4} \sqrt{9 \pm \sqrt{57}}$ & $\pm \frac{9}{4 \sqrt{2}} \sqrt{33 \pm \sqrt{753}}$ & $\pm \frac{3}{\sqrt{2}} \sqrt{33 \pm 27}$ \\
\hline
\end{tabular}

\section{Summary and discussion}

We have derived a general formula for the representation matrix of the operator $\hat{D}(n)$ on sets of four-valent spin network states corresponding to a link configuration $(p, q, r, s)$, with fixed $x$, where $x=\operatorname{Min}\left\{s, \frac{1}{2}(-p+q+r+s)\right\}$. This expression, formula (3.3), is given in terms of eight matrices whose entries have to be computed for each $x$. The spin network states are not independent, but obey certain linear relations, the 
Mandelstam constraints. The linearly dependent states have to be identified explicitly, otherwise one obtains spurious eigenvalues after diagonalization. At this moment, the only restriction on obtaining the full spectrum on four-valent spin networks is computing capacity. It may be possible that the formulae obtained above for $x=1,2,3$ can be written in a more symmetric form that can be generalized to arbitrary $x$; so far we have not been able to do this.

We have observed that eigenvalues occur in pairs of opposite sign. Thus, for odd $x+1$ there is a zero-eigenvalue for an eigenstate that does not vanish modulo the Mandelstam constraints. This means that there exist four-valent spin networks "without volume" (which by construction are non-planar; the planar ones are all annihilated by $\hat{\mathcal{V}}$ because of antisymmetry). Another consequence is that after taking the modulus, as is necessary for constructing the local volume operator $\hat{\mathcal{V}}(n)$, all non-zero eigenvalues are (at least) two-fold degenerate.

The generalization of our results to spin networks of higher valence is algebraically more complicated; in this case already the counting of spin networks is less straightforward. Still, there are no obvious obstructions to deriving analogues of our matrix formula (3.3).

As for the geometric interpretation of our eigenvalue expressions, we have seen that their dependence on the occupation numbers $(p, q, r, s)$ is not particularly simple, for example, they do not just depend on the total number of links, $(p+q+r+s)$. One possibility is to try to extract from them a certain asymptotic behaviour, say, as the total number of links becomes very large. For example, for fixed $x$, one may look at spin networks corresponding to link configurations of the form $(p, p, p, x)$, where $p$ runs through all odd or even integers, depending on whether $x$ is odd or even. For the cases studied in the last section, one finds the following asymptotic behaviour of the eigenvalues of $\hat{D}(n)$ for large $p$ and to highest order in $p$ :

$$
\begin{array}{ll}
x=1: & \sim \pm \frac{3 \sqrt{3}}{2^{5}} p^{2}, \\
x=2: & \sim \pm \frac{3 \sqrt{3}}{2^{4}} p^{2} \\
x=3: & \sim \pm \frac{3 \sqrt{3}}{2^{5}}(2 \pm 1) p^{2} .
\end{array}
$$

The "local volume", $\hat{\mathcal{V}}(n)$, therefore increases linearly in $p$, and can become arbitrarily large for the classes of spin networks considered here. Such asymptotic relations may be useful in estimating the contributions of certain sectors of the Hilbert space of spin network states in numerical approximations.

\section{Acknowledgements}

I am grateful to the members of the Max-Planck-Institut for their kind hospitality. 


\section{References}

[1] J.B. Baez, Spin network states in gauge theory, to appear in Adv. Math., e-Print Archive: gr-qc 941 1007; Spin networks and non-perturbative quantum gravity, to appear in the proceedings of the AMS Short Course on Knots and Physics, e-Print Archive: gr-qc 9504036.

[2] C. Rovelli and L. Smolin, Nucl. Phys. B 442 (1995) 593.

[3] R. Loll, Phys. Rev. Lett. 75 (1995) 3048.

[4] R. Loll, Nucl. Phys. B 444 (1995) 619.

[5] L. Smolin, personal communication.

[6] A. Ashtekar, personal communication; Polymer geometry at Planck scale and quantum Einstein equations, preprint Penn. State Univ. (1995).

[7] J.F. Barbero, Phys. Rev. D 51 (1995) 5498, 5507.

[8] J. Kogut and L. Susskind, Phys. Rev. D 11 (1975) 395;

J.B. Kogut, Rev. Mod. Phys. 55 ( 1983) 775.

[9] R. Loll, Nucl. Phys. B 368 (1992) 121; B 400 (1993) 126. 